\title{
Viscoelastic Evaluation of Different Hyaluronic Acid Based Fillers Using Vibrational Optical Coherence Tomography
}

\author{
Frederick H. Silver ${ }^{1,2 *}$, Ruchit G. Shah ${ }^{3}$, Nikita Kelkar2, \\ Dominick Benedetto4, Dale DeVore ${ }^{5}$, Justin Cohen ${ }^{6}$
}

\author{
${ }^{1}$ Department of Pathology and Laboratory Medicine, Robert Wood Johnson Medical School, Rutgers, The State University of New \\ Jersey, Piscataway, NJ, USA \\ ${ }^{2}$ OptoVibronex, LLC, Mt. Bethel, Pa, USA \\ ${ }^{3}$ Graduate Program in Biomedical Engineering, Rutgers, The State University of New Jersey, Piscataway, NJ, USA \\ ${ }^{4}$ Center for Advanced Eye Care, Vero Beach, FL, USA \\ ${ }^{5}$ DV Consulting, Chelmsford, MA, USA \\ ${ }^{6}$ Glasgold Group, Princeton, NJ, USA \\ Email: *fhsilver@hotmail.com
}

How to cite this paper: Silver, F.H., Shah, R.G., Kelkar, N., Benedetto, D., DeVore, D. and Cohen, J. (2019) Viscoelastic Evaluation of Different Hyaluronic Acid Based Fillers Using Vibrational Optical Coherence Tomography. Materials Sciences and Applications, 10, 423-431.

https://doi.org/10.4236/msa.2019.105031

Received: April 18, 2019

Accepted: May 24, 2019

Published: May 27, 2019

Copyright $\odot 2019$ by author(s) and Scientific Research Publishing Inc. This work is licensed under the Creative Commons Attribution International License (CC BY 4.0).

http://creativecommons.org/licenses/by/4.0/

\begin{abstract}
Hylauronic acid (HA) is used as a viscoelastic in Ophthalmology during cataract surgery based on its high viscosity at rest, its ability to shear thin and dissipate energy during phacoemulsification. However, these properties of HA solutions would make them susceptible to migration when used as dermal filler materials. In this study, we apply a new technique termed vibrational optical coherence tomography (VOCT) to compare the physical properties of different HA solutions and fillers used in facial aesthetics. Results presented in this study suggest that HA solutions and HA dermal fillers have markedly different physical properties. HA solutions are highly viscoelastic with high \% viscous losses while fillers tend to have lower viscous energy dissipation properties. Clinical observations suggest that the high loss fillers are injected more superficially in the face where tension and internal and external forces are more likely minimized giving tissue of the hands and lips more volume and allowing more natural movement. In contrast, the lower loss gels that are used to lift tissue, generally have a higher G', and are injected deeper into the face where injection and internal forces are likely to be higher. It is concluded that HA filler gel design can be optimized by use of VOCT to evaluate the \% viscous energy loss both in vitro and in vivo.
\end{abstract}

\section{Keywords}

Hyaluronic Acid, Injectable Filler, Dermal Filler, Plastic Surgery, Cosmetic 
Surgery, Viscoelastic, Mechanical Properties, Facial Surgery, Optical Coherence Tomography, Vibrational Optical Coherence Tomography

\section{Introduction}

Hyaluronic acid (HA) also known as hyaluronan, is polysaccharide composed of repeating $\beta$-1-4-linked $\mathrm{D}$-glucuronic acid and $\beta$-1-3-linked $\mathrm{N}$-acetyl-D-glucosamine disaccharide units [1]. The various names of HA reflect the properties of the molecule under various conditions. At neutral $\mathrm{pH}, \mathrm{HA}$ exists as a polyelectrolyte with associated cations, frequently as a sodium salt; therefore, the name sodium hyaluronate. The name was later amended to "hyaluronate" in reference to its salt form or "hyaluronan," a term used to encompass all forms of the molecule [1].

HA is found ubiquitously in the ECM of all vertebrate tissues including blood, synovial fluid, vitreous body, pericellular matrix, cytoplasm, and nucleus. Its use as a viscoelastic in Ophthalmology during cataract surgery is based on the molecule's ability to shear thin and thereby absorb energy during phacoemulsification [2]. This property is a result of the reversible hydrogen bonding that occurs between side chains of the molecule [2]. However, to limit its ability to shear thin and flow under applied stress requires the formation of covalent crosslinks between HA chains.

Hyaluronic acid (HA) injectable dermal fillers (DFs) have become the most popular agents for soft tissue contouring and volumizing. HA fillers are reported to be characterized by ideal properties such as biocompatibility, biodegradability, and versatility. These filler properties enable HA DFs to dominate and revolutionize the filler market with numerous products differing in HA sourcing, degree of crosslinking, concentration, hardness, cohesiveness, and consistency. The inclusion or lack of inclusion of an anesthetic, indications for use, and longevity of correction are other variables examined in designing DFs [3] [4]. However, there are potential complications that can arise from use of these materials [5] [6].

The rheological and mechanical properties of HA fillers have been reviewed recently [4]. HA fillers have been developed that are both viscous solutions and solution-gel mixtures containing uncrosslinked and crosslinked macromolecules. HA fillers are crosslinked using a number of different crosslinking agents. The HA concentration of fillers ranges from 5.5 to $28 \mathrm{mg} / \mathrm{ml}$, \% crosslinking from 0 to $20 \%$, shear elastic modulus from 100 to $1800 \mathrm{~Pa}$, and particle size from 0 to over 1050 micrometers [4]. Observations of the physical properties of HA solutions and gels are important parameters that control the behavior of fillers in clinical use. However, currently, there are no tests available to study the physical properties of fillers both before and after implantation. We have developed a new technique termed vibrational optical coherence tomography (VOCT) that 
can be used to evaluate HA filler properties both in vitro and in vivo.

The purpose of this paper is to introduce the use of VOCT to evaluate the physical properties of HA fillers before clinical use. VOCT has been used to evaluate the properties of skin and scar tissue in vivo [7]-[14], it can also be used to evaluate the mechanical behavior of skin after filler injections. Characterization of filler viscoelastic properties is needed to determine the desired clinical outcome; how the starting HA filler properties influence the quality of the outcome is a question that needs to be answered.

\section{Materials and Methods}

VOCT is new technique that measures the resonant frequency and viscous losses of solutions of macromolecules and gels. Unlike measurement of solution viscosity or viscoelasticity using a viscometer, this technique provides measures of both cohesive as well as viscous energy dissipation of materials when a mechanical vibration is applied. This method can be used both in vitro and in vivo unlike a viscometer which can only be used in vitro [7]-[14].

Commercial samples of pure HA with molecular weights of $5 \mathrm{k}$ and $1.8 \mathrm{M}$ were obtained from Lifecore Biomedical, LLC. (Chaska, Mn). Samples of dermal fillers listed in Table 1 were obtained from Allergan Inc. (Dublin, Ire). A drop containing between 0.1 and $0.2 \mathrm{ml}$ of each sample was placed on a glass slide for examination. The slide was the placed on a rigid frame that had a hole cut out so that the sound was applied to the slide from below. The sample volume did not influence the measurement of resonant frequency. Samples were tested at $22^{\circ} \mathrm{C}$ by applying a sinusoidal sound wave from a speaker placed beneath the sample as discussed previously [7]-[14]. The frame, speaker, and glass slide resonant frequencies were measured in the absence of the samples. Sample weighted displacements were corrected for any resonant frequencies of the speaker and support

Table 1. HA formulations studied using vibrational optical coherence tomography. The $\%$ viscous loss is reported at $30 \mathrm{~Hz}$ for the HAs studied.

\begin{tabular}{|c|c|c|c|c|}
\hline Sample & Conc (mg/ml) & Resonant Freq $(\mathrm{Hz})$ & Viscous Loss (\%) & Clinical Use \\
\hline Restylane D & 20 & 150 & 27 & $\mathrm{md} / \mathrm{deep}$ \\
\hline Restylane Lft & 20 & 140 & 27 & $\mathrm{md} /$ deep \\
\hline Voluma XC & 20 & 150 & 25 & $\mathrm{md} /$ deep \\
\hline Vollure XC & 17.5 & 150 & 35 & sup/md/deep \\
\hline Restylane L & 20 & 150 & 35 & sup/md/deep \\
\hline Juvederm U & 24 & $140-180$ & 40 & $\mathrm{md} / \mathrm{sup}$ \\
\hline Restylane S & 20 & 180 & 40 & sup \\
\hline $\mathrm{HA}(5 \mathrm{k})$ & 20 & 140 & 60 & N/A \\
\hline $\mathrm{HA}(1.8 \mathrm{M})$ & 20 & 220 & 60 & N/A \\
\hline
\end{tabular}

Note: the standard deviation of resonant frequency measurements is about $6 \mathrm{~Hz}$ while that for \% viscosity measurements is about $10 \%$ of the $\%$ reported. Abbreviations: $\mathrm{sp}=$ superficial, $\mathrm{md}=\mathrm{mid}, \mathrm{N} / \mathrm{A}=$ not applicable, Freq = resonant frequency, Conc = concentration . 
materials, or from line voltage variations.

Transverse forces were applied to the sample by positioning an acoustic loudspeaker (Intervox S225RA-40) beneath the sample. A function generator (Agilent) was used to drive the speaker with sinusoidal waveforms at varying amplitudes and frequencies. The resonant frequency was determined as the frequency at which the maximum displacement was observed [7]-[14].

Transverse sample displacement was measured by spectral-domain optical coherence tomography (SD-OCT), a non-contact, interferometric technique [7]-[14]. The resonant frequency of each sample was initially estimated at a single point by measuring the transverse displacement resulting from sinusoidal driving frequencies ranging from $20 \mathrm{~Hz}$ to $500 \mathrm{~Hz}$, in steps of $50 \mathrm{~Hz}$. Once the region where the maximum displacement was identified, smaller steps of $10 \mathrm{~Hz}$ were used to more accurately identify the peak frequency and the actual resonant frequency, $f_{n \text {. }}$

Measurement of Elastic and Viscous Behaviors

The elastic and viscous components of the viscoelastic behavior were obtained from measurements made from the driving frequency peak as described previously [7]-[14]. The elastic component was obtained from the peak height while the viscous component was obtained by dividing the change in frequency at the half height of the peak (i.e. $3 \mathrm{db}$ down from maximum peak in power spectrum) by the driving frequency. This method is known as the half-height bandwidth method discussed by Paul Macioce (http://www.roush.com/wp-content/uploads/2015/09/Insight.pdf). The viscous loss in percent for each sample was tabulated as a function of the applied sound frequency.

\section{Results}

Weighted displacement versus frequency plots for droplets of pure HA with molecular weights $5 \mathrm{k}$ and $1.8 \mathrm{M}$ are shown in Figure 1. Major peaks are shown at frequencies of $140 \mathrm{~Hz}(\mathrm{Mw}=5 \mathrm{k})$ and $220 \mathrm{~Hz}(\mathrm{Mw}=1.8 \mathrm{M})$. The $\%$ viscous contribution to the viscoelastic behavior is shown in Figure 2. Both pure HA samples, independent of molecular weight, have viscous losses that are about $60 \%$ at $30 \mathrm{~Hz}$ suggesting that these materials are more viscous than elastic at low frequencies. At frequencies above $200 \mathrm{~Hz}$, these HAs have \% viscous losses that approach $10 \%$ and behave almost purely elastically. In the absence of crosslinks, HA molecules are highly mobile and do not remain in place when an applied force is exerted on their surfaces.

Weighted displacement measurements versus frequency for representative dermal fillers listed in Table 1 are shown in Figure 3 and the \% loss behavior for typical dermal fillers examined in this study are shown in Figure 4 . The weighted displacement versus resonant frequency curves for all the filler materials exhibit resonant frequencies that center around $150 \mathrm{~Hz}$. The percent loss behaviors of the fillers fall into the two groups the group with losses from about $35 \%$ to 
$40 \%$ at $20 \mathrm{~Hz}$ (group A) and those with losses of about $25 \%$ (group B) at $20 \mathrm{~Hz}$. At high frequencies the \% loss decreases to about $5 \%$ for all the dermal filler samples studied. At high frequencies all fillers behave almost purely elastic. Note the gel dermal fillers that are used in cosmetic surgery are more elastic (less

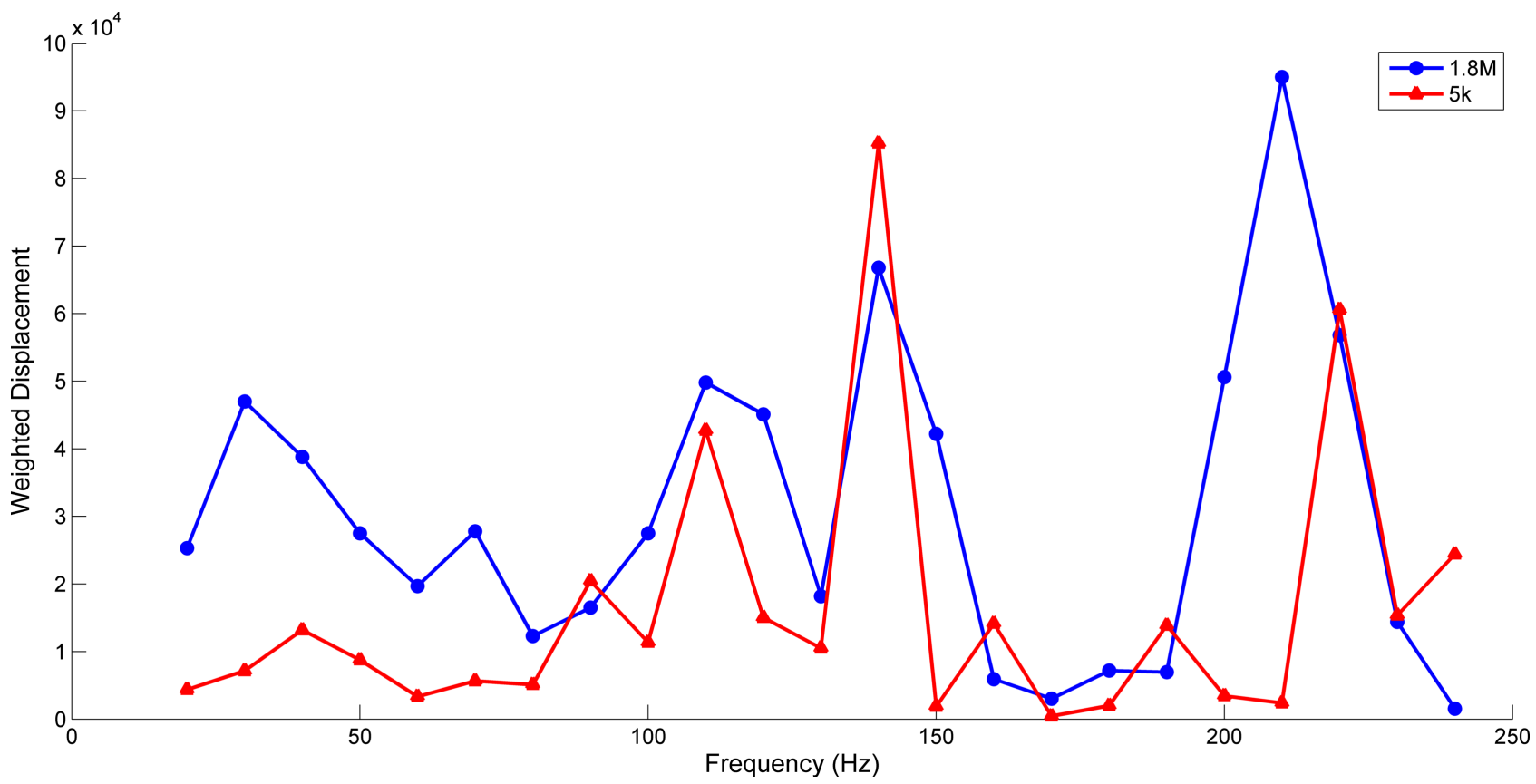

Figure 1. Plot of weighted displacement versus frequency for a drop of purified HA solution with molecular weights of $5 \mathrm{~K}$ and 1.8 M. The major peaks are seen at $140 \mathrm{~Hz}(\mathrm{Mw}=5 \mathrm{k})$ and $220 \mathrm{~Hz}(\mathrm{Mw}=1.5 \mathrm{M})$ for a drop of solution on a glass slide. Note the non-major peaks arise from multiple vibrations associated with within the droplet that occur during energy dissipation.

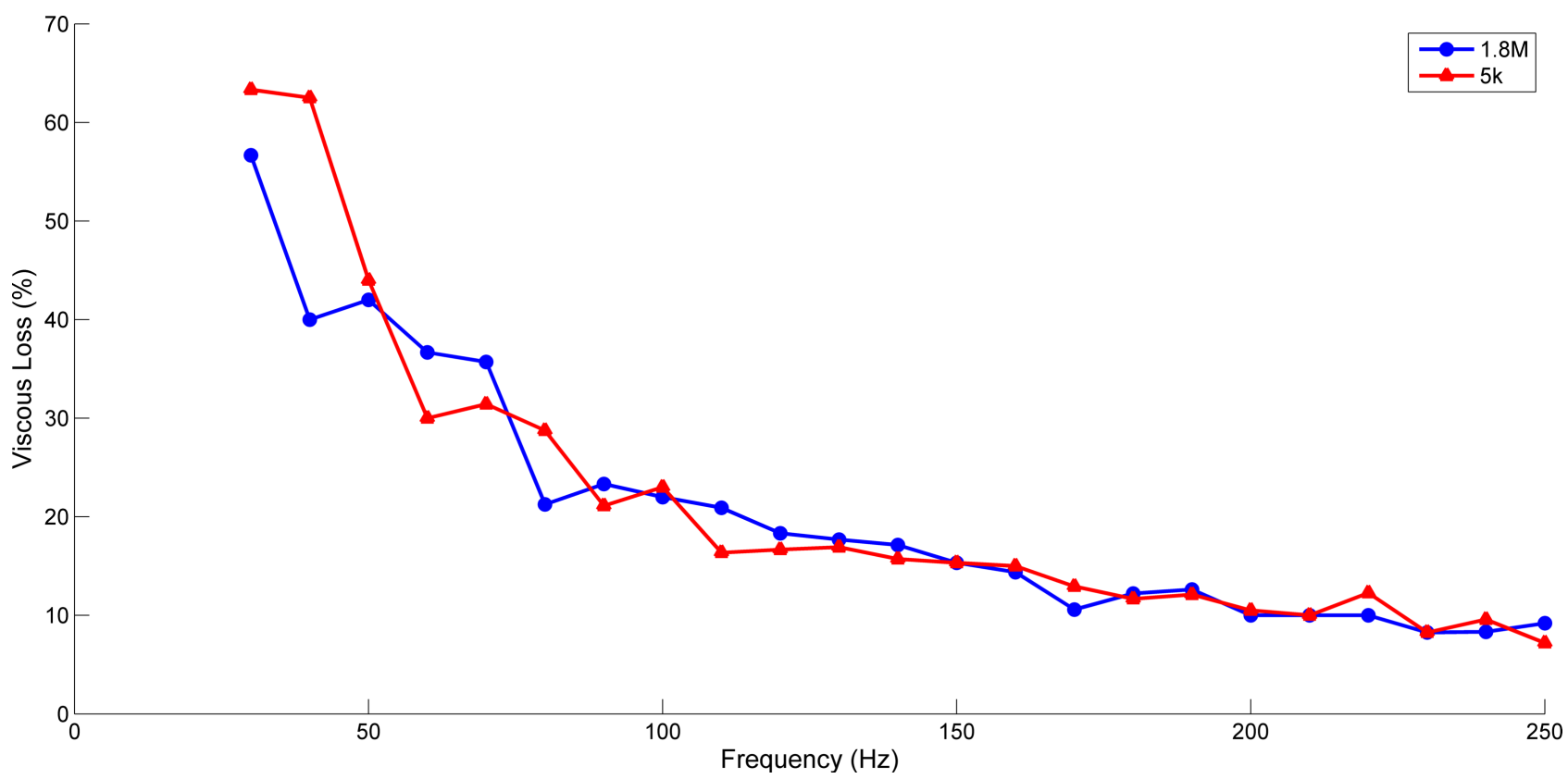

Figure 2. \% Viscous loss as a function of frequency for a drop of purified HA solutions. Note the \% viscous loss of low (5 $\mathrm{k}$ ) and high $(1.8 \mathrm{M})$ molecular weight HA fractions are very high at low frequency $(60 \%)$ and approach a value of $10 \%$ at high frequencies. At low frequencies HA solutions are viscous liquids that dissipate energy by shear thinning. 


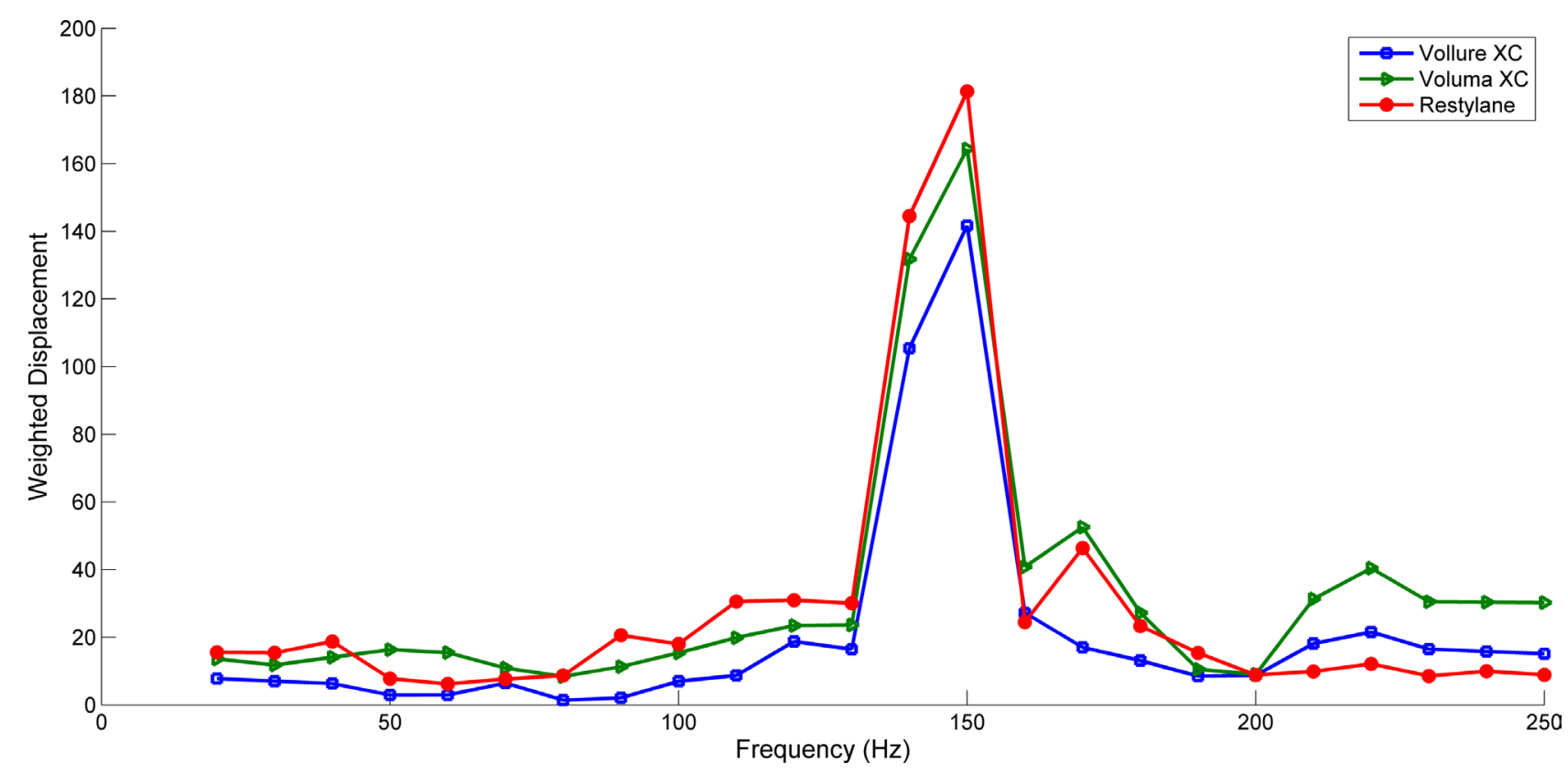

Figure 3. Typical plot of weighted displacement versus frequency for dermal fillers. Note all dermal fillers had major peaks and resonant frequencies at about $150 \mathrm{~Hz}$ with several subpeaks being present. Restylane data shown in the figure is for Restylane L.

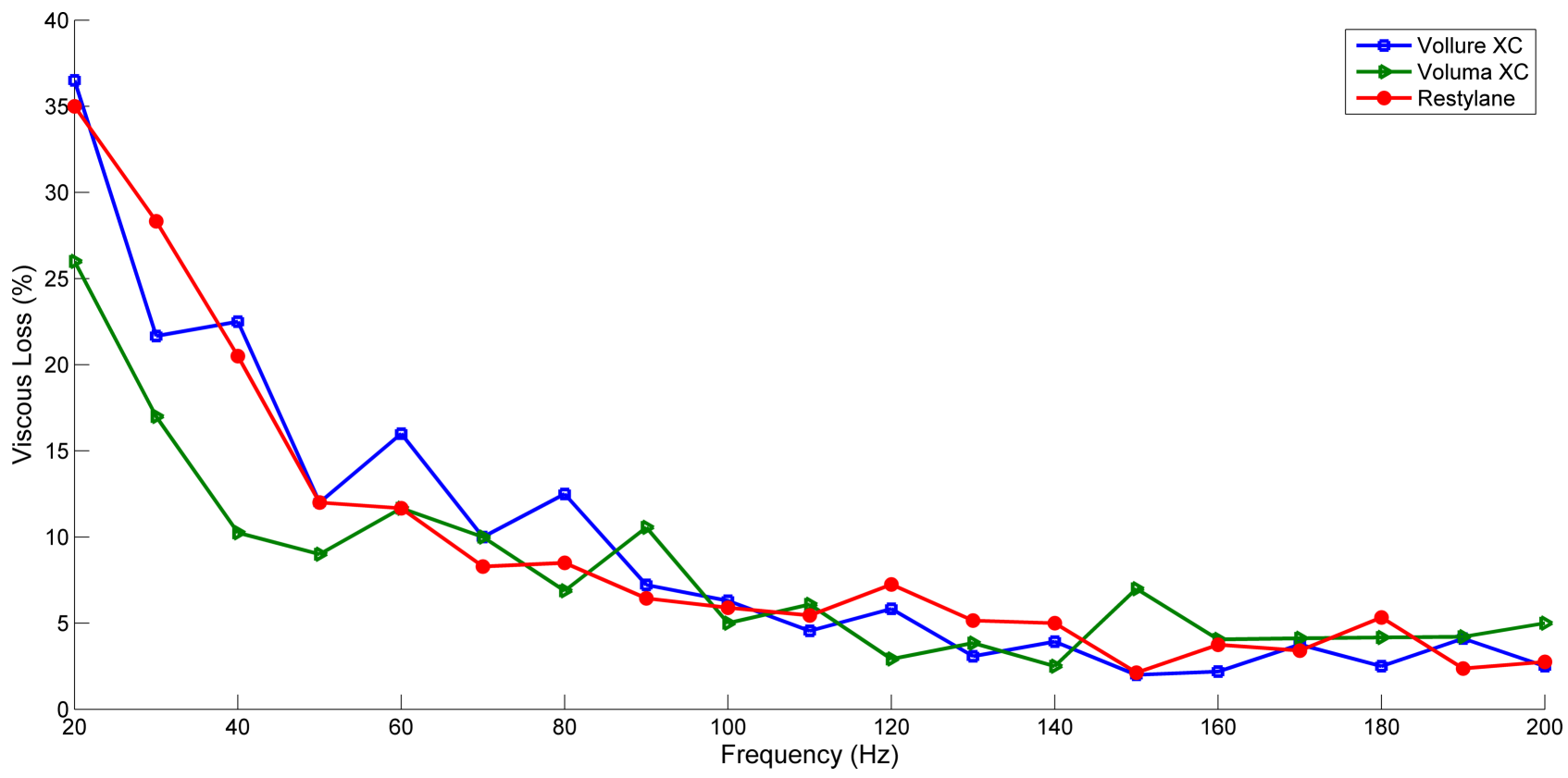

Figure 4. Typical \% viscous loss as a function of frequency for dermal fillers. Note the difference between high loss fillers and low loss fillers. The high loss group includes Juvederm Ultra, Restylane L, Vollure XC, Restylane Silk. This group had \% viscous losses in the 35\% - 40\% range at $20 \mathrm{~Hz}$ while the low loss group Voluma XC, Restylane Define, Restylane Lyft and had viscous losses of about $25 \%$ at $20 \mathrm{~Hz}$. Restylane data shown in the figure is for Restylane L.

viscous) at high frequencies than are HA liquid solutions.

The clinical observations of the uses of the different dermal fillers are shown in Table 1. It is observed that group B dermal fillers (Restylane Define, Restylane Lyft, Voluma XC), the low loss fillers at low shear rates, are clinically used in the mid to deep areas of the skin whereas group A dermal fillers, the high loss fillers 
(Juvederm Ultra, Restylane L, Restylane Refine and Restylane Silk, and Vollure $\mathrm{XC})$, are generally used more superficially.

\section{Discussion}

HA solutions containing polymeric molecules of different molecular weights are biocompatible natural materials that are viscoelastic and shear thin at high shear rates [3]. While the biocompatibility of these solutions is an important property, the viscoelasticity and liquid-like behavior reflected by the high energy loss at low frequencies limits their ability to remain in place as filler materials. Therefore modifications must be made to solutions of viscoelastics used in Ophthalmology to make these materials function as dermal fillers that do not migrate after injection.

The most important findings reported in this study, suggest that the viscous behavior of pure HA solutions is much higher than that obtained with any of the dermal filler materials. While pure HA solutions of low and high molecular weights exhibit multiple resonant frequencies, it is clear that uncrosslinked, non-particulate containing HA solutions independent of molecular weight behave as highly viscoelastic liquids. The high \% loss at low frequencies indicates that these materials will absorb energy at low shear rates, dissipating energy as needed in cataract surgery. Then at high shear rates they act in an elastic fashion and can be removed from the eye via their cohesive forces as a bolus of material. In contrast they make poor fillers since they will dissipate energy by migrating in an irreversible fashion when an external force is applied to tissue.

In contrast, HA dermal filler materials are required to remain in place after injection even after the application of external forces that occur during sleeping or other daily activities. The addition of crosslinks and resulting gel particles decreases the shear thinning of HA and reduces the energy losses and viscoelasticity of the filler materials. HA dermal fillers can be classified into two groups, based on the results reported in this paper: the groups are characterized by differences in the viscous losses at low strain rates. The high loss group includes Juvederm Ultra, Restylane L, Vollure XC, Restylane Silk. This group had \% viscous losses in the $35 \%$ - 40\% range while the low loss group Voluma XC, Restylane Define, Restylane Lyft and had viscous losses of about $25 \%$.

Clinically, the high loss "thinner" gel group has been observed to be more appropriate for superficial indications to remove fine wrinkles and for lip enhancement. Tissue tension due to forces from collagen fibers along Langer's lines and external forces are more likely to be minimized in these regions of the face. In contrast, the lower loss "thicker" DF gels that are used to lift tissue, generally have a higher G', and are injected deeper into the face where volume induced internal forces are likely to be higher. Optimization in the physical properties of the injectable dermal fillers evaluated in this study would be difficult to achieve without a multifactorial design that considers concentration, particle size, and degree of crosslinking in a single study. Differences in these factors 
make analysis of the optimization of filler composition and properties difficult to achieve.

\section{Conclusions}

HA solutions containing polymeric molecules of different molecular weights are biocompatible natural materials that are viscoelastic and shear thin at high shear rates. While pure HA solutions of low and high molecular weights exhibit multiple resonant frequencies, it is clear that uncrosslinked non-particulate containing HA solutions independent of molecular weight flow easily under applied stress that makes them poor filler materials to use for tissue augmentation. The addition of crosslinks and gel particles in HA filler materials decreases the shear thinning of HA and reduces the energy losses and viscoelasticity.

Our results suggest that HA fillers can be categorized into two groups. Clinically, the high loss group has been observed to be used more superficially in the face where tissue tension and internal and external forces are more likely minimized. In contrast, the lower loss gels that are used to lift tissue, generally have a higher G', and are injected deeper into the face where injection and internal forces are likely to be higher. Unlike HA dermal filler gels, HA solutions used in cataract surgery exhibit high \% viscous losses and are not useful in aesthetic surgery due to their tendency to migrate under applied internal and external forces. Future work will evaluate the \% viscous losses of dermal filler materials after injection in vivo.

\section{Conflicts of Interest}

The authors declare no conflicts of interest regarding the publication of this paper.

\section{References}

[1] Dicker, K.T., Gurski, L.A., Pradhan-Bhat, S., Witt, R.L., Farach-Carson, M.C. and Jia, X. (2014) Hyaluronan: A Simple Polysaccharide with Diverse Biological Functions. Acta Biomaterialia, 10, 1558-1570. https://doi.org/10.1016/j.actbio.2013.12.019

[2] Silver, F.H. and Benedetto, D. (1996) Polysaccharides Used in Ophthalmology. In: Dumitriu, S., Ed., Polysaccharides Used in Medicinal Applications, Marcel Dekker Inc., New York, 689-703.

[3] Fallacara, A., Manfredini, S., Durini, E. and Vertuani, S. (2017) Hyaluronic Acid Fillers in Soft Tissue Regeneration. Facial Plastics Surgery, 33, 87-96. https://doi.org/10.1055/s-0036-1597685

[4] Greene, J.J. and Sidle, D.M. (2015) The Hyaluronic Acid Fillers Current Understanding of the Tissue Device Interface. Facial Plastic Surgery Clinics of North America, 23, 423-432. https://doi.org/10.1016/j.fsc.2015.07.002

[5] Urdiales-Gálvez, F., Escoda Delgado, N., Figueiredo, V., Lajo-Plaza, J.V., Mira, M., Mar Mira, Ortíz-Martí, F., del Rio-Reyes, R., Romero-Á lvarez, N., Ruiz del Cueto, S., Martía A. Segurado, M.A. and Villanueva Rebenaque, C. (2017) Preventing the Complications Associated with the Use of Dermal Fillers in Facial Aesthetic Procedures: An Expert Group Consensus Report. Aesthetic Plastic Surgery, 41, 667-677. 
https://doi.org/10.1007/s00266-017-0798-y

[6] Vedamurthy, M. (2018) Beware What You Inject: Complications of Injectables-Dermal Fillers. Journal of Cutaneous and Aesthetic Surgery, 11, 60-66. https://doi.org/10.4103/JCAS.JCAS_68_18

[7] Shah, R., DeVore, D. and Pierce, M.G. (2016) Morphomechanics of Dermis: A Method for Non-Destructive Testing and Collagenous Tissues. Skin Research and Technology, 23.

[8] Shah, R., Pierce, M.C. and Silver, F.H. (2017) A Method for Non-Destructive Mechanical Testing of Tissues and Implants. Journal of Biomedical Materials Research Part A, 105, 5-22. https://doi.org/10.1002/jbm.a.35859

[9] Silver, F.H. and Silver, L.L. (2017) Non-Invasive Viscoelastic Behavior of Human Skin and Decellularized Dermis Using Vibrational OCT. Dermatology Clinics \& Research, 3, 174-179.

[10] Shah, R.G., DeVore, D. and Silver, F.H. (2018). Biomechanical Analysis of Decellularized Dermis and Skin: Initial in Vivo Observations Using OCT and Vibrational Analysis. JJournal of Biomedical Materials Research Part A, 106.

https://doi.org/10.1002/jbm.a.36344

[11] Silver, F.H., DeVore, D. and Shah, R. (2017) Biochemical, Biophysical and Mechanical Characterization of Decellularized Dermal Implants. Material Sciences and Applications, 8, 873-888. https://doi.org/10.4236/msa.2017.812064

[12] Silver, F.H. and Shah, R.G. (2018) Mechanical Spectroscopy and Imaging of Skin Components in Vivo: Assignment of the Observed Moduli. Skin Research and Technology, 25. https://doi.org/10.1111/srt.12594

[13] Silver, F.H. and Silver, L.L. (2018) Use of Vibrational Optical Coherence Tomography in Dermatology. Archives of Dermatology and Skin Care, 1, 3-8.

[14] Silver, F.H., Shah, R.G., Benedetto, D., Dulur, A. and Kirn, T. (2019) Virtual Biopsy and Physical Characterization of Tissues, Biofilms, Implants and Viscoelastic Liquids Using Vibrational Optical Coherence Tomography. World Journal Mechanics, 9, 1-6. https://doi.org/10.4236/wjm.2019.91001 